RESEARCH H IGHLIGHTS

\section{Orchestrating T-cell development}

$\mathrm{T}$ cells fall into two lineages $-\alpha \beta$ $\mathrm{T}$ cells and $\gamma \delta \mathrm{T}$ cells - and these are thought to develop independently in the thymus. But, a new study from Adrian Hayday's group shows that $\mathrm{CD} 4^{+} \mathrm{CD}^{+}$(double positive, $\mathrm{DP}$ ) thymocytes, which are currently thought of simply as progenitors of $\alpha \beta$ T cells, regulate the development of early progenitors and of $\gamma \delta$ T cells.

A previous study from this group used transcriptional profiling to define a set of genes that is associated with $\gamma \delta \mathrm{T}$ cells. Surprisingly, the expression of a subset of these genes was found to depend on the presence of DP thymocytes. In mice that lack normal DP cells, such as mice deficient in the pre-T-cell receptor (TCR) $\alpha$-chain, $\gamma \delta$-biased gene expression by double-negative 2 (DN2) and DN3 cells was reduced, and this was associated with impaired function of peripheral $\gamma \delta \mathrm{T}$ cells.

So, how do DP T cells affect maturing $\gamma \delta \mathrm{T}$ cells and early progenitors? DP cells were found to express the transcription factor ROR- $\gamma \mathrm{t}$ (retinoic-acid-receptor-related orphan receptor- $\gamma \mathrm{t}$ ) and to produce lymphotoxin (LT). These are features of lymphoid-tissue-inducer cells; LT is a cytokine that binds the LT receptor (LTR) and has a role in mediating the organization of lymphoid tissues. To test whether the $\gamma \delta$ biased gene set is regulated by LT, the authors cultured fetal lobes from mice deficient in TCR- $\beta$ (which lack normal DP cells) with an agonist antibody specific for the LTR, and they showed that this rescued the expression of the $\gamma \delta$-biased gene set. In addition, a soluble LT- $\beta$ R-Fc fusion protein disrupted expression of this gene set in wild-type thymocytes, and consistent with this, expression of the gene set is partially impaired in mice deficient in either LT or the LTR.

LT is generally thought to act on stromal cells by inducing expression of adhesion molecules that mediate tissue reorganization. However, in the thymic cortex, where DN and DP cells are found, epithelial cells are not known to respond to LT, indicating the possibility that DP cells might act directly on thymocytes rather than indirectly through stromal cells. In support of this, the authors showed that, in the absence of stroma, early thymocyte progenitors and $\gamma \delta$ thymocytes are directly responsive to DP cells and the LTR agonist antibody although this experiment also showed that LT-mediated signalling is necessary but not sufficient to induce expression of the genes in question. The contribution of other factors produced by DP cells to the differentiation of early thymocyte progenitors and $\gamma \delta$ thymocytes remains to be clarified.

This study reveals that DP cells influence the development of other thymocytes, indicating that there is a biology of integrated T-cell activities that we have little appreciation of at present.

Elaine Bell

\section{(2) References and links} ORIGINAL RESEARCH PAPER Silva-Santos, B. Pennington, D. J. \& Hayday, A. C. Lymphotoxinmediated regulation of $\gamma \delta$ cell differentiation by $\alpha \beta$ T cell progenitors. Science 9 Dec 2004 (doi:10.1126/science.1103978).

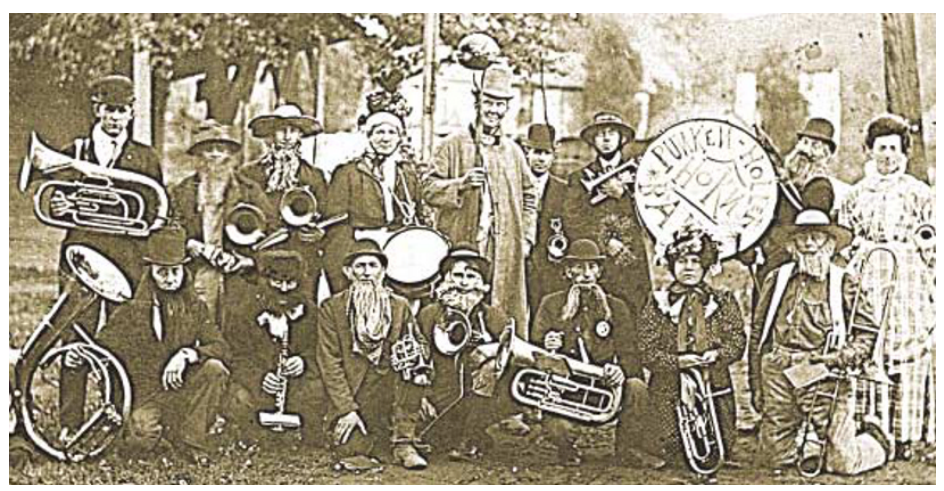

\section{IN BRIEF}

\section{TRANSPLANTATION}

Heart transplantation in baboons using $\alpha 1,3$-galactosyltransferase gene-knockout pigs as donors: initial experience.

Kuwaki, K. et al. Nature Med. 11, 29-31 (2005).

Marked prolongation of porcine renal xenograft survival in baboons through the use of $\alpha 1,3$-galactosyltransferase gene-knockout donors and the cotransplantation of vascularized thymic tissue.

Yamada, K. et al. Nature Med. 11, 32-34 (2005).

Natural antibodies specific for $\alpha 1,3$-galactose (Gal), which is present on the surface of all pig cells, are the main cause of hyperacute xenograft rejection in non-human primates. Cardiac and renal transplants from $\alpha 1,3$-galactosyltransferase geneknockout pigs, which do not express Gal, have extended survival in baboons, with a maximum of 83 days for the renal transplant and 179 days for the cardiac transplant. These are the longest recorded xenograft survival times of this type.

\section{HIV}

The influence of CCL3L1 gene-containing segmental duplications on HIV-1/AIDS susceptibility.

Gonzalez, E. et al. Science 6 Jan 2005 (doi:10.1126/science.1101160).

Variation between individuals in the copy number of CCchemokine ligand 3 (CCL3)-like 1 (CCL3L1), a duplicated isoform of CCL3, has a significant effect on immunity to HIV. Individuals with a lower than average CCL $3 L 1$ copy number have an increased risk of becoming infected with HIV and of progressing rapidly to AIDS, and vice versa. High levels of CCL3L1, which is the most potent known ligand for the HIV co-receptor CCR5, are thought to block the association between CCR 5 and HIV gp 120 required for virus internalization. Individuals with a low CCL3L1 copy number were more susceptible to the disease-accelerating effects of detrimental CCR5 genotypes, supporting the crucial involvement of CCR5 and its ligands in determining HIV pathogenesis.

\section{ANTIGEN PROCESSING}

Endogenous MHC class II processing of a viral nuclear antigen after autophagy.

Paludan, C. et al. Science 9 Dec 2004 (doi:10.1126/science.1104904).

Although MHC class II molecules mainly present peptides of exogenous origin, some peptides are derived from endogenous proteins. Paludan et al. showed that inhibition of lysosomal acidification leads to accumulation of the endogenous antigen Epstein-Barr virus nuclear antigen 1 (EBNA1) in cytosolic vesicles. Some of these vesicles also contained a lysosomal marker, and most had ultrastructural features of autophagosomes. Consistent with this, interferon- $\gamma$ production by EBNA1-specific $\mathrm{CD} 4^{+} \mathrm{T}$ cells was decreased after stimulation with either cells treated with an inhibitor of autophagy or cells in which expression of the essential autophagy gene Atg12 had been reduced. The authors suggest that lysosomal processing after autophagy allows MHC-class-IIrestricted immune surveillance of some endogenous proteins. 